SFB

Stabilität von

Diversifikationseffekten im

Markowitz-Modell

Nicolai Bissantz, Verena Steinorth, Daniel Ziggel

Nr. 26/2010

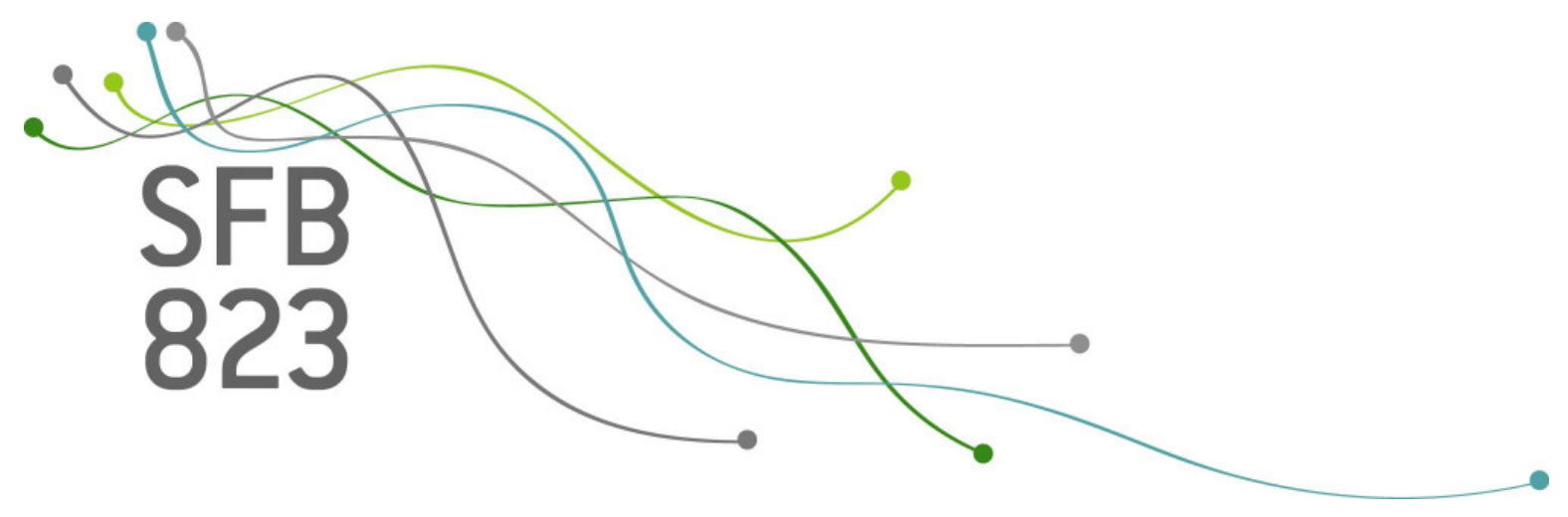





\title{
Stabilität von Diversifikationseffekten im Markowitz-Modell
}

\author{
Von \\ Dr. Nicolai Bissantz, Bochum, Verena Steinorth, Münster und Dr. Da- \\ niel Ziggel, Münster
}

\section{Zusammenfassung}

Im Zuge der Finanzkrise wurde deutlich, dass das Risiko in klassischen Modellen zur Portfoliotheorie deutlich unterschätzt wurde. Die Instabilität der relevanten Risikoparameter, also Korrelationen und Volatilitäten, führte dazu, dass Diversifikationseffekte über- und somit Risiken unterschätzt wurden. Ziel dieses Beitrags ist es, Diversifikationseffekte in verschiedenen Marktphasen des letzten Jahrzents zu untersuchen und die Auswirkungen für das Risikomanagement zu quantifizieren. Dabei zeigt sich, dass sich die Parameter bzw. Risiken sehr deutlich mit der jeweiligen Marktphase ändern und eine Parameterschätzung aus historischen Mittelwerten nicht zielführend ist.

JEL - Klassifikation: C 52, G 11, G 32

JEL - Schlüsselwörter: Modellevaluierung, Portfoliooptimierung, Risikomanagement

\section{Anschriften der Autoren}

Dr. Nicolai Bissantz, Ruhr-Universität Bochum, Fakultät für Mathematik, Mathematik III, Universitätsstraße 150, 44780 Bochum, nicolai.bissantz@rub.de, +49/234/32/23291, Verena Steinorth, quasol GmbH, Marktallee 8, 48165 Münster, Dr. Daniel Ziggel, quasol GmbH, Marktallee 8, 48165 Münster 



\section{Stabilität von Diversifikationseffekten im Markowitz-Modell}

\section{Einleitung}

Die effektive Zusammenstellung von Wertpapieren in einem Portfolio bzw. die Porfoliooptimierung sind zentrale Fragestellungen sowohl für Finanzunternehmen als auch für Privatpersonen. Dabei besteht bei der strategischen Asset Allocation die grundlegende Aufgabenstellung darin, ein bestimmtes Vermögen optimal auf verschiedene Anlageklassen aufzuteilen. Hier sind die zu erwartende Rendite und das mögliche (Verlust-) Risiko die entscheidenden Parameter. Als Durchbruch, aus wissenschaftlicher Sicht, kann die Arbeit von Markowitz (1952) betrachtet werden, für welche er 1990 den Wirtschaftsnobelpreis erhielt. So soll ein Investor nur in effiziente Portfolios investieren, das heißt Portfolios, die bei einer vorher vom Investor als Ziel gesetzten Rendite das kleinste Risiko besitzen bzw. bei einem vorher vom Investor als noch akzeptabel festgelegten Risiko die höchste Rendite. Dabei wird das Risiko des Portfolios durch die Varianz bzw. Standardabweichung seines Gesamtwerts bestimmt. Hierbei können Korrelationen zwischen den einzelnen Assets dazu führen, dass das Risiko des gesamten Portfolios im Gegensatz zur Investition in einzelne Anlageklassen deutlich gesenkt werden kann.

In zahlreichen Arbeiten wurde nachgewiesen, dass die strategische Asset Allocation den Großteil der Performance einer Anlage beeinflusst. Brinson/Hood/Beebower (1986) und Brinson/Singer/Beebower (1991) beziffern den Einfluss auf $90 \%$ bis $94 \%$, während Ibbotson/Kaplan (2000) Werte zwischen $82 \%$ und $88 \%$ nennen. Dies zeigt die Bedeutung der strategischen Asset Allocation. Andere Faktoren wie Timing oder Strategieumsetzung spielen hingegen nur eine untergeordnete Rolle.

Entscheidend für eine sinnvolle Wahl des Portfolios und damit für den späteren Anlageerfolg ist eine verlässliche Schätzung der relevanten Parameter (Rendite, Varianz, Korrelationen), wobei in verschiedenen Studien gezeigt wurde, dass beim Markowitz-Ansatz die Rendite den mit Abstand wichtigsten Parameter darstellt. So kamen Chopra und Ziemba (1993) zum Ergebnis, dass für mittlere Risikotoleranzen falsche Renditeschätzer eine 
11 mal höhere Auswirkung haben als falsche Risikoschätzer. Kallberg und Ziemba (1984) sowie Schäfer und Zimmermann (1998) zeigten ebenfalls, dass die Schätzprobleme beim Markowitz-Ansatz hauptsächlich die Rendite betreffen.

Aktuelle Entwicklungen an den Finanzmärkten rücken jedoch die Risikoperspektive wieder stärker in der Vordergrund. Während der Finanzkrise stiegen Volatilitäten ${ }^{1}$ und Korrelationen sprunghaft an, was sich in deutlich erhöhten Risikokennzahlen widerspiegelte. Dieses Phänomen wird von Zimmermann/Drobetz/Oertmann (2002) als "Correlation Breakdown“ beschrieben. Offensichtlich besteht in Krisenzeiten eine positive Korrelation zwischen Volatilitäten und Korrelationen von verschiedenen Assetklassen und der Diversifikationsgedanke greift gerade dann nicht, wenn er eigentlich vor Verlusten schützen soll.

In dieser Arbeit werden die Auswirkungen von variierenden Parametern in verschiedenen Marktphasen empirisch untersucht und für verschiedene Beispiele quantifiziert. Dazu werden die resultieren Risikokennzahlen für unterschiedliche Marktphasen verglichen. Somit lassen sich Rückschlüsse über die Stabilität von Diversifikationseffekten in der klassischen Portfoliotheorie ziehen. Es zeigt sich, dass Risiken deutlich unterschätzt werden, insbesondere wenn historische Mittelwerte als Parameterschätzer verwendet werden. Daher müssen alternative Modelle und Methoden eingeführt werden, um eine verlässliche Diversifikation zu gewährleisten.

\section{Klassische Portfoliotheorie}

Das von Markowitz (1952) entwickelte Modell kann als Meilenstein in der Entwicklung von modernen Theorien in den Bereichen Asset Allocation und Portfoliooptimierung betrachtet werden. Dabei wurde angenommen, dass $N$ Anlagen mit normalverteilter Rendite $r_{i}$ für die $i$-te Anlage existieren. Ziel ist dabei, die Portfoliogewichte $\left(\omega_{1}, \omega_{2}, \cdots, \omega_{N}\right)$ optimal zu wählen, wobei $\omega_{i}$ den Anteil darstellt, der in Anlage $i$ investiert wird. Auch heutzutage ist das Markowitz-Modell in der Praxis noch weit verbreitet und wird von vielen Anlegern zur Optimierung von Portfolios verwendet. Dabei muss in vielen Anwendungen gelten $\omega_{i} \geq 0$ und $\sum_{i=1}^{N} \omega_{i}=1$. Die entscheidenden Parameter für die Zusammenstellung des Portfolios sind

\footnotetext{
${ }^{1}$ In dieser Arbeit wird die Volatilität als Standardabweichung des jeweiligen Wertpapiers definiert.
} 
die erwartete Rendite des Portfolios $\left(r_{P}\right)$ und das Risiko des Portfolios, welches über die Standardabweichung $\left(\sigma_{P}\right)$ definiert ist.

Aus der Markowitz-Theorie folgt, dass die für Investitionen interessanten Portfolios sogenannte „effiziente Portfolios“ sind, d.h. Portfolios deren Kombination $\left(r_{P}, \sigma_{P}\right)$ von keinem anderen Portfolio mit kleinerer Standardabweichung bei gleicher Rendite bzw. mit höherer Rendite bei gleicher Standardabweichung dominiert wird. In der Praxis wird einer der beiden Werte vorgegeben und dann das Portfolio so gewählt, dass der zweite Parameter optimiert wird. Somit muss entweder eine Mindestrendite $r_{\text {min }}$ oder eine maximale Volatilität $\sigma_{\max }$ vorgegeben werden. Es ergeben sich also die folgenden Optimierungsprobleme:

\section{(OP1)}

$$
\begin{array}{ll}
\min & \sigma_{P} \\
\text { so dass } \quad r_{P} \geq r_{\min }
\end{array}
$$

\section{(OP1')}

$\begin{array}{ll} & \max \quad r_{P} \\ \text { so dass } & \sigma_{P} \leq \sigma_{\max } .\end{array}$

Ein wesentlicher Vorteil dieses Vorgehens besteht in der automatischen Berücksichtigung von Korrelationseffekten zwischen den verschiedenen Wertpapieren, die dazu führen können, dass Investitionen in auf den ersten Blick ungünstig erscheinende Anlagen zu einer Senkung des PortfolioGesamtrisikos führen. Dazu betrachte man z.B. ein Portfolio, das sich zu $80 \%$ aus einem Wertpapier mit einer Renditeerwartung von $5 \%$ bei einer Volatilität von $3 \%$ zusammensetzt und zu $20 \%$ aus einem riskanteren Wertpapier mit einer Renditerwartung von 10\% bei einer Standardabweichung von $6 \%$. Es ergibt sich somit unmittelbar eine erwartete Portfoliorendite von $6 \%$. Die Portfolio-Gesamtvolatilität beträgt im Falle von Unkorreliertheit der beiden Wertpapier nur 2.7\%. Bei einer Korrelation von -0.5 sogar nur $2.4 \%$, d.h. das Portfolio erreicht eine höhere Renditeerwartung als das sicherere der beiden Wertpapiere, bei einem deutlich kleineren Risiko als jedes einzelne von ihnen, wenn das Risiko durch die Standardabweichung gemessen wird. Eine Illustration der Auswirkung verschiedener Korrelationen und Mischungsverhältnisse $\left(\omega_{i} \in[0,1]\right)$ der beiden Wertpapiere gibt Abbildung 1, in der die Renditen und Volatilitäten der Portfolios für beliebige Mischungsverhältnisse dargestellt sind. 


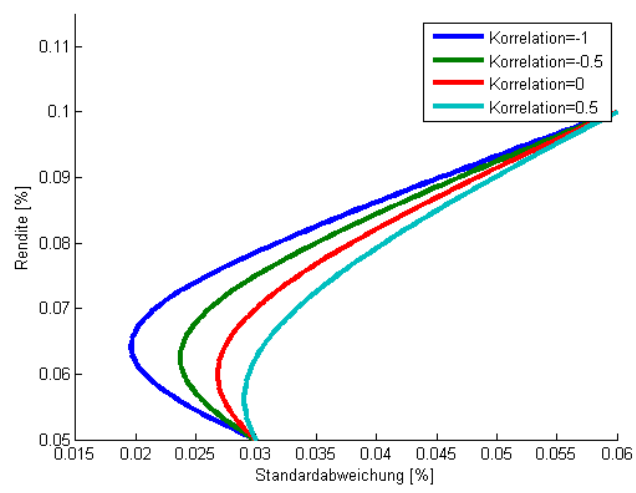

Abbildung 1: Effizienzlinien für Portfolios aus zwei Wertpapieren mit Rendite und Standardabweichung von $(5 \%, 3 \%)$ bzw. $(10 \%, 6 \%)$ für verschiedene Werte der Korrelation zwischen den beiden Wertpapieren.

In diese Modellrechnung, basierend auf der Markowitz-Theorie, gehen allerdings entscheidend die Annahmen ein, dass die Renditen normalverteilt sind bzw. die Parameter der Wertpapiere (Rendite, Korrelation und Standardabweichung) verlässlich geschätzt werden können - und dass diese sich nicht während der Anlageperiode ändern. In den letzten Jahren zeigte sich jedoch immer deutlicher, dass eine verlässliche Parameterschätzung nur sehr schwer möglich ist. Einerseits herrscht inzwischen Gewissheit über eine deutliche Zeitabhängigkeit von Korrelationen und Varianzen, im wesentlichen in der Form, dass Varianzen und Korrelationen dazu neigen zu steigen, wenn die Märkte fallen bzw. zu fallen, wenn die Märkte steigen. Anderseits gibt es klare Anzeichen für starke Abhängigkeiten zwischen Varianzen und Korrelationen, die von Frennberg und Hansson (1993), Zimmermann/Drobetz/Oertmann (2002) oder Andersen/Bollerslev/Diebold/Ebens (2001) dargestellt wurden.

\section{Correlation Breakdown}

In Diskussionen über die Bedeutung von Korrelationen und Volatilitäten für das Risikomanagement und das Hedging fiel in den letzten Jahren ver- 
mehrt der Begriff „Correlation Breakdown“. Dieser beschreibt das Phänomen, dass sich Korrelationen und Volatilitäten zwischen ruhigen, gewöhnlichen und hektischen, unüblichen Marktphasen unterscheiden. So zeigte sich, dass die Parameter in turbulenten Marktphasen ansteigen und eine starke positive Beziehung zwischen den Korrelationen und den Standardabweichungen besteht. Daher werden Diversifikationseffekte genau dann überschätzt, wenn sie am meisten benötigt werden, also in turbulenten Marktsituationen. Zudem macht es das sich fortlaufend ändernde Korrelationsmuster sehr schwierig, eine optimale Risikostrategie zu wählen.

Der Börsencrash im Oktober 1987 und die Finanzkrise im Jahr 2008 zeigen deutlich, dass die Korrelationsstruktur sehr sensibel auf extreme Marktgeschehnisse reagiert. In beiden Situationen stiegen die Korrelationen erheblich und blieben für einen bestimmten Zeitraum auf diesem hohen Niveau bestehen.

Um den Börsen-Crash 1987 war ein deutlicher Korrelationsanstieg zu beobachten. Meric/Meric (1997) bestätigen diesen Sachverhalt aus europäischer Sicht. Die durchschnittliche Korrelation zwischen 13 europäischen Aktienmärkten stieg von 0,37 in der Periode vor dem Crash 1987 auf 0,50 in der Periode danach. Ähnliche Ergebnisse finden sich bei Rey (2000). So stiegen die durchschnittlichen Korrelationen, die von Januar 1973 bis Dezember 1986 und von Januar 1988 bis Dezember 1999 gemessen wurden, von 0,40 auf 0,55. Dabei wurden Daten aus der Schweiz, den USA, Großbritannien, Kanada, Deutschland, Italien, Frankreich und Japan verwendet. Beschränkt man sich auf den Oktober 1987, so betrug die durchschnittliche Korrelation zwischen den internationalen Aktienmarktrenditen laut Rey (2000) sogar 0,68. Desweiteren bestätigen die Untersuchungen von Longin und Solnik (1995), dass die Korrelationen in Zeiten hoher Volatilität generell steigen und die Beziehung zwischen den Korrelationen und den Volatilitäten umso ausgeprägter ist, je höher die Volatilität ist.

Diese Ergebnisse zwingen Investoren dazu den Diversifikationsgedanken zu hinterfragen. So brechen die Strukturen, die eigentlich für die Reduzierung des Gesamtrisikos verwendet werden, gerade dann zusammen, wenn die Märkte fallen. Gerade im letzten Jahrzehnt, in welchem zwei große Krisen an den Finanzmärkten auftraten, ist zu hinterfragen, ob die klassische Portfoliotheorie noch zu verlässlichen Risikoschätzern führt. 


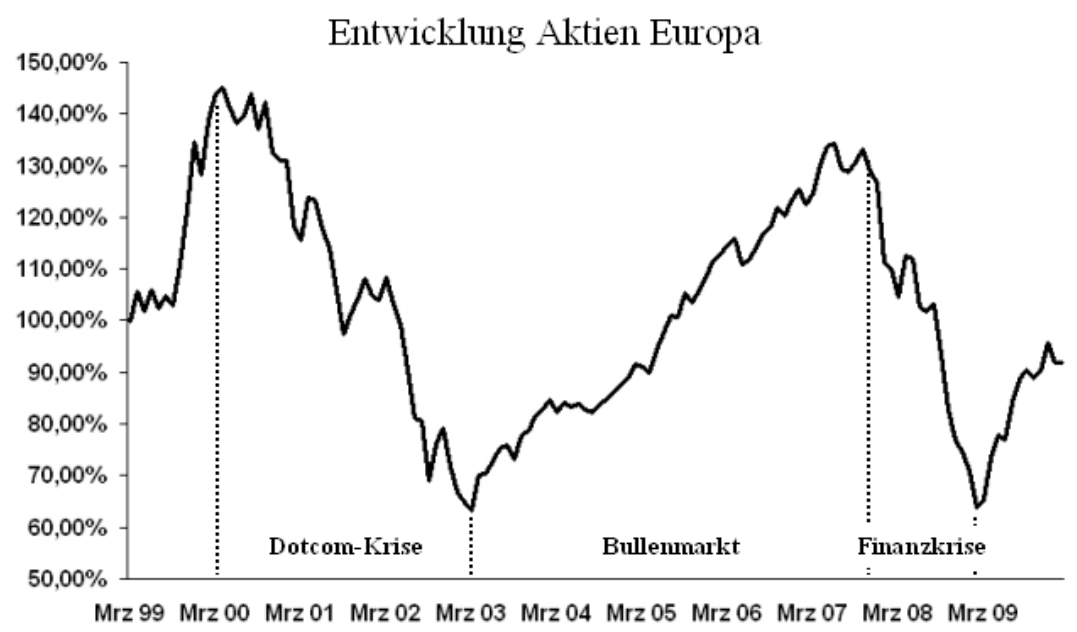

Abbildung 2: Schematische Darstellung der Unterteilung des Analysezeitraums am Beispiel der Entwicklung des EURO STOXX 50 - Total Return Index.

\section{Empirische Untersuchung}

Im folgenden Abschnitt wird untersucht, wie sich Korrelationsstrukturen und Volatilitäten in verschiedenen Phasen des letzten Jahrzehnts entwickelt haben. Dabei wird zwischen vier verschiedenen Zeiträumen unterschieden. So umfasst der gesamte Analysezeitraum die Periode 31.03.1999 bis 26.02.2010. Innerhalb dieses Zeitraums werden zusätzlich zwei Bärenmärkte (Dotcom-Krise ${ }^{2}$, Finanzkrise ${ }^{3}$ ) und ein Bullenmarkt ${ }^{4}$ isoliert betrachtet. Abbildung 2 illustriert die zeitliche Abfolge der Phasen.

\section{Datenbasis}

Wir verwenden Monatsendwerte repräsentativer Indizes, um die Kennzahlen der jeweiligen Assetklassen zu bestimmen. Lediglich bei den Immo-

\footnotetext{
${ }^{2} 31.03 .2000$ bis 31.03 .2003

${ }^{3} 30.04 .2008$ bis 31.03 .2009

${ }^{4} 30.04 .2003$ bis 31.03 .2008
} 
bilien wird ein offener Immobilienfonds für die Berechnungen herangezogen, da kein repräsentativer Index für Immobilien vorliegt. Insbesondere werden folgende Indizes bzw. Assetklassen in der Untersuchung berücksichtigt:

- EURO STOXX 50 - Total Return Index (Aktien Europa)

- MSCI USA - Total Return Index (Aktien USA)

- MSCI Japan - Total Return Index (Aktien Japan)

- MSCI Emerging Markets - Price Index (Aktien EM)

- REX - Total Return Index (Staatsanleihen)

- iBoxx EUR Corporates AA - Total Return Index (Corporates AA)

- iBoxx EUR Corporates BBB - Total Return Index (Corporates BBB)

- Euribor 3 Monate - Offered Rate (Geldmarkt)

- HFRI Fund of Funds Composite Index - Total Return Index (Hedgefonds)

- CRB Spot Index - Price Index (Rohstoffe)

- UniImmo: Deutschland - Total Return Index (Immobilien)

Insgesamt umfasst der Analysezeitraum 11 Jahre, also 132 Monate. Eine längere Historie ist nicht verfügbar, da durch die Einführung des Euro verschiedene neue Indizes entstanden sind.

\section{Berechnung der Kennzahlen}

Die Berechnung der erwarteten Renditen und der Risiken eines Portfolios beruht auf Schätzungen der entsprechenden Kennzahlen für die relevanten Perioden. Wir betrachten für jede einzelne Assetklasse $a(a \in\{1, \ldots, 11\})$ stetige Monatsrenditen. Diese werden durch

$$
r_{a}(j)=\ln \left(\frac{\text { Indexstand zum Ende des } \mathrm{j} \text {-ten Monats }}{\text { Indexstand zum Ende des }(\mathrm{j}-1) \text {-ten Monats }}\right)
$$


berechnet, wobei $j$ den vorliegenden Zeitraum der Daten monatsweise durchnummeriert. Als durchschnittlich zu erwartende Jahresrendite ${ }^{5}$ ergibt sich somit $\hat{R}_{a}(J)=12 \cdot \overline{r_{a}(J)}$, wobei $\overline{r_{a}(J)}$ den Mittelwert der monatlichen Renditen in der jeweiligen Periode darstellt. Die zugehörigen Monate werden durch die Indexmenge $J$ zusammengefasst.

Aus den Renditen $r_{a}(j)$ für Anlageklasse $a$ ergibt sich als Schätzer für die Varianz der Renditen

$$
\hat{\sigma}_{a}^{2}(J)=12 \cdot\left[\frac{1}{n-1} \sum_{j \in J}\left(r_{a}(j)-\overline{r_{a}(J)}\right)^{2}\right],
$$

wobei $n$ die Anzahl der Monate ist, die zur jeweiligen Periode gehören. Die Volatilität errechnet sich als Wurzel der Varianz. Analog ergeben sich als Schätzer für die Korrelation zwischen zwei Anlageklassen $a$ und $b(a, b \in$ $\{1, \ldots, 11\})$

$$
\hat{\rho}_{a, b}(J)=\frac{12}{n-1} \sum_{j \in J}\left(\frac{r_{a}(j)-\overline{r_{a}(J)}}{\sqrt{\hat{\sigma}_{a}^{2}(J)}}\right) \cdot\left(\frac{r_{b}(j)-\overline{r_{b}(J)}}{\sqrt{\hat{\sigma}_{b}^{2}(J)}}\right)
$$

und als Schätzer für die zugehörige Kovarianz

$$
\hat{\sigma}_{a, b}^{2}(J)=\sqrt{\hat{\sigma}_{a}^{2}(J) \cdot \hat{\sigma}_{b}^{2}(J)} \cdot \hat{\rho}_{a, b}(J) .
$$

Aus der geschätzten Rendite $\hat{R}_{a}(J)$ und Varianz $\hat{\sigma}_{a}^{2}(J)$ ergibt sich eine parametrische Schätzung des 99\%-Value-at-Risk einer Anlageklasse mit dem 1\%-Quantil der Standardnormalverteilung $q_{0.01}=-2.326$ als

$$
\operatorname{VaR}_{a}(99 \%)(J)=\hat{R}_{a}(J)-2.326 \cdot \sqrt{\hat{\sigma}_{a}^{2}(J)} .
$$

Der $\operatorname{VaR}_{a}(99 \%)$ kann somit in eine Komponente $\operatorname{VaR}_{a}(99 \%)_{\text {ew }}$ zerlegt werden, die sich aus der erwarteten Rendite (bzw. dem entsprechenden Schätzer) ergibt, sowie einer ,stochastischen“ Komponente $\operatorname{VaR}_{a}(99 \%)_{\text {stoch }}=$ $-2.326 \cdot \sqrt{\hat{\sigma}_{a}^{2}(J)}$, die sich aus den (geschätzten) Volatilitäten berechnet. Wie im folgenden Abschnitt deutlich wird, beeinflussen die Korrelationen den Value-at-Risk eines Portfolios ebenfalls, da sie für die Berechnung der Volatilität des Gesamtportfolios benötigt werden.

${ }^{5}$ Zur Vereinfachung der Interpretation der folgenden Berechnungen und der resultierenden Ergebnisse sind sämtliche Kennzahlen in dieser Arbeit, insbesondere Volatilitäten und Value-at-Risks, auf eine einjährige Anlageperiode skaliert. 
Mit Hilfe der geschätzten Parameter der einzelnen Anlageklassen ist es möglich die Rendite und das Risiko eines Portfolios mit den Gewichten $\left(\omega_{1}, \ldots, \omega_{N}\right)$ in einer Periode $J$ zu berechnen. So ergibt sich unmittelbar als erwartete Rendite

$$
\hat{r}_{P}(J)=\sum_{a=1}^{N} \omega_{a} \hat{R}_{a}(J),
$$

sowie als Varianz

$$
\hat{\sigma}_{P}^{2}(J)=\sum_{a, b=1}^{N} \omega_{a} \omega_{b} \hat{\sigma}_{a, b}^{2}(J) .
$$

Schließlich ergibt sich der 99\%-Value-at-Risk VaR $(99 \%)_{P(J)}$ des Portfolios in einem Zeitraum $J$ zu

$$
\operatorname{VaR}(99 \%)_{P(J)}=\hat{r}_{P}(J)-2.326 \cdot \sqrt{\hat{\sigma}_{P}^{2}(J)} .
$$

Die stochastische Komponente ist somit

$$
\operatorname{VaR}(99 \%)_{\text {stoch }}=-2.326 \cdot \sqrt{\hat{\sigma}_{P}^{2}(J)} .
$$




\section{Parameter in den verschiedenen Marktphasen}

Tabelle 1 und 2 stellen die Volatilitäten und Korrelation für die einzelnen Marktphasen zusammen. Daraus wird deutlich, wie stark diese Parameter in der Zeit schwanken. Während dieser Effekt in der Dotcom-Krise lediglich Aktien betraf, erfasst er in der Finanzkrise beinahe alle Assetklassen. Vergleicht man die Werte innerhalb des Bullenmarktes mit denen der Finanzkrise, so zeigt sich, dass die Volatilitäten von Aktien, Rohstoffen und Unternehmensanleihen um den Faktor 2 bis 3 anstiegen. Lediglich Staatsanleihen, Immobilien und den Geldmarkt betraf diese Entwicklung nicht. Aber auch der Vergleich der Kennzahlen des gesamten Zeitraums und der Finanzkrise zeigt noch große Differenzen.

Betrachtet man die durchschnittlichen Korrelationen zu allen anderen Wertpapieren, so ergibt sich ein ähnliches Ergebnis. Die Korrelationen von Aktien, Rohstoffen und Unternehmensanleihen niedrigerer Bonität stiegen deutlich an. Daher konnten Diversifikationseffekte lediglich durch die gleichzeitige Investition in sichere Anlagen erzielt werden. Bei diesen sanken die Korrelationen sogar teilweise recht deutlich. So kommt es, dass die durchschnittliche Korrelation über alle Indizes in allen Phasen sehr stabil ist.

Werden Korrelationen zwischen einzelnen Assetklassen verglichen, so verstärken sich die Ergebnisse noch. Beispielsweise stieg die Korrelation zwischen Aktien aus Japan und den USA von 0,45 während des Bullenmarktes auf 0,88 in der Finanzkrise. Noch ausgeprägter war die Veränderung zwischen Rohstoffen und Aktien. Hier stieg die Korrelation zwischen Rohstoffen und Aktien aus Europa von -0,12 auf 0,56. Im Gegenzug sanken die Korrelationen zwischen sicheren und riskanteren Anlagen. Beispielsweise nahm die Korrelation zwischen Staatsanleihen und Rohstoffen von 0,13 auf -0,55 ab. ${ }^{6}$

Die Ergebnisse zeigen, dass Diversifikationseffekte zwischen ähnlichen Anlageklassen, z.B. innerhalb von Aktien verschiedener Länder, überschätzt werden. Somit sind die tatsächlich eingegangenen Risiken deutlich höher als erwartet. Eine große Diversifikation wird nur bei gleichzeitiger Investition in sehr unterschiedliche Anlageklassen erreicht. Besonders die Beimischung von Anlageklassen, die als relativ sicher gelten, ist unbedingt notwendig.

\footnotetext{
${ }^{6}$ Die vollständigen Korrelationsmatrizen finden sich unter www.quasol.de/publikationen.html
} 
Tabelle 1

Volatilitäten in verschiedenen Marktphasen

\begin{tabular}{lcccc}
\hline Index / Phase & Gesamt & Dotcom & Bullenmarkt & Finanzkrise \\
\hline Aktien Europa & $17,39 \%$ & $19,30 \%$ & $11,15 \%$ & $21,22 \%$ \\
Aktien USA & $16,30 \%$ & $18,64 \%$ & $9,17 \%$ & $27,11 \%$ \\
Aktien Japan & $18,02 \%$ & $15,33 \%$ & $14,58 \%$ & $31,71 \%$ \\
Aktien EM & $20,99 \%$ & $20,91 \%$ & $15,80 \%$ & $33,09 \%$ \\
Staatsanleihen & $3,14 \%$ & $2,80 \%$ & $3,03 \%$ & $4,40 \%$ \\
Corporates AA & $3,57 \%$ & $3,22 \%$ & $2,99 \%$ & $5,49 \%$ \\
Corporates BBB & $4,54 \%$ & $4,03 \%$ & $2,66 \%$ & $8,35 \%$ \\
Geldmarkt & $0,34 \%$ & $0,21 \%$ & $0,27 \%$ & $0,35 \%$ \\
Hedgefonds & $6,08 \%$ & $5,00 \%$ & $4,32 \%$ & $8,99 \%$ \\
Rohstoffe & $11,90 \%$ & $9,29 \%$ & $7,88 \%$ & $26,20 \%$ \\
Immobilien & $1,14 \%$ & $0,59 \%$ & $1,56 \%$ & $0,43 \%$
\end{tabular}

Tabelle 2

Durchschnittliche Korrelationen in verschiedenen

Marktphasen

\begin{tabular}{lcccc}
\hline Index / Phase & Gesamt & Dotcom & Bullenmarkt & Finanzkrise \\
\hline Aktien Europa & 0,18 & 0,07 & 0,07 & 0,36 \\
Aktien USA & 0,24 & 0,13 & 0,12 & 0,39 \\
Aktien Japan & 0,2 & 0,13 & 0,08 & 0,38 \\
Aktien EM & 0,25 & 0,14 & 0,16 & 0,36 \\
Staatsanleihen & $-0,06$ & $-0,02$ & 0,05 & $-0,17$ \\
Corporates AA & 0,11 & 0,06 & 0,11 & 0,11 \\
Corporates BBB & 0,26 & 0,23 & 0,16 & 0,33 \\
Geldmarkt & $-0,17$ & 0,00 & $-0,09$ & $-0,21$ \\
Hedgefonds & 0,05 & 0,05 & $-0,04$ & 0,03 \\
Rohstoffe & 0,17 & 0,02 & 0,05 & 0,27 \\
Immobilien & $-0,01$ & 0,09 & 0,00 & $-0,16$ \\
Durschnitt & 0,11 & 0,08 & 0,15 & 0,06
\end{tabular}




\section{Auswirkungen der Parameteränderungen auf Risikokennzahlen realer Portfolios}

Um die Auswirkungen der veränderten Parameter zu veranschaulichen und genauer zu quantifizieren werden die Risikokennzahlen vier verschiedener Portfolios für die jeweiligen Phasen bestimmt. Die große Variabilität der Korrelationen und Volatilitäten führt zu einem stark variierenden stochastischen Beitrag $\left(\mathrm{VaR}_{\text {stoch }}\right)$ zum gesamten Value-at-Risk $(\mathrm{VaR})$. Im Folgenden werden wir uns auf die Betrachtung dieser stochastischen Komponente beschränken, da ihre Änderungen auf die Variabilität der Korrelationen und Volatilitäten zurückzuführen ist. Daneben repräsentiert diese Größe den Diversifikationseffekt, der im Portfolio erzielt werden kann. Es zeigt sich, dass diese Komponente eine wesentliche Änderung des gesamten VaR bewirkt.

Bei einem der betrachteten Portfolios handelt es sich um das naiv diversifizierte Portfolio, also das Portfolio, bei dem alle Indizes den gleichen Anteil am Gesamtportfolio ausmachen. Daneben werden zwei Fonds für Privatanleger (Deka Zukunftsplan I, Deka-Euroland Balance TF) untersucht. Zusätzlich wird ein mögliches Musterportfolio institutioneller Investoren, wie es sich bei Sparkassen oder Volksbanken in der Depot-A Steuerung findet, analysiert (vgl. Otto (2008)). Die genauen Aufteilungen der Portfolios finden sich in Tabelle 3. Zur Vereinfachung wurde angenommen, dass die im Portfolio enthaltenen Assetklassen perfekt durch den jeweiligen Index abgebildet werden. Weiterhin wird eine Anlagesumme von 100.000.000 Euro angenommen, um den Value-at-Risk auch in Euro angeben zu können.

Tabellen 4-7 geben die stochastische Komponente des VaR für die einzelnen Portfolios wider. Die Ergebnisse zeigen deutlich, dass allein durch die Änderung der Parameter Korrelation und Volatilität der Value-at-Risk drastisch verändert wird. So verdreifacht sich die Value-at-Risk $V_{a} R_{\text {stoch }}$ für das naiv diversifizierte Portfolio, wenn die Parameter des Bullenmarktes durch die Parameter der Finanzkrise ausgetauscht werden. Aber auch während der Dotcom-Krise war das Risiko ca. 50\% höher als im Bullenmarkt. Analoges gilt für die Fonds Deka Zukunftsplan I und Deka-Euroland Balance TF. Besonders für letzteren ist dieses Ergebnis überaschend, da es sich durchaus um einen breit diversifizierten Fond handelt.

Für das institutionelle Portfolio sind die Auswirkungen nicht so groß. Durch den sehr hohen Anteil an sicheren Anlagen, insbesondere Staats- 
Tabelle 3

Portfoliogewichte

\begin{tabular}{lcccc}
\hline Index / Portfolio & Naiv & Zukunftsplan & Euroland Balance & Institutionell \\
\hline Aktien Europa & $9,09 \%$ & $54,00 \%$ & $58,70 \%$ & $1,70 \%$ \\
Aktien USA & $9,09 \%$ & $34,00 \%$ & $0,00 \%$ & $1,70 \%$ \\
Aktien Japan & $9,09 \%$ & $7,80 \%$ & $0,00 \%$ & $0,50 \%$ \\
Aktien EM & $9,09 \%$ & $2,20 \%$ & $0,00 \%$ & $0,25 \%$ \\
Staatsanleihen & $9,09 \%$ & $0,00 \%$ & $20,00 \%$ & $75,40 \%$ \\
Corporates AA & $9,09 \%$ & $0,00 \%$ & $4,00 \%$ & $6,00 \%$ \\
Corporates BBB & $9,09 \%$ & $0,00 \%$ & $2,80 \%$ & $4,50 \%$ \\
Geldmarkt & $9,09 \%$ & $2,00 \%$ & $14,50 \%$ & $5,45 \%$ \\
Hedgefonds & $9,09 \%$ & $0,00 \%$ & $0,00 \%$ & $1,00 \%$ \\
Rohstoffe & $9,09 \%$ & $0,00 \%$ & $0,00 \%$ & $1,00 \%$ \\
Immobilien & $9,09 \%$ & $0,00 \%$ & $0,00 \%$ & $2,50 \%$
\end{tabular}

Tabelle 4

$\mathrm{VaR}_{\text {stoch }}$ Naive Diversifikation

\begin{tabular}{lcc}
\hline Phase / VaR & In $\%$ & In Euro \\
\hline Gesamt & $-15,37 \%$ & $-14,2$ Mio. Euro \\
Dotcom & $-13,40 \%$ & $-12,5$ Mio. Euro \\
Finanzkrise & $-29,18 \%$ & $-25,3$ Mio. Euro \\
Bullenmarkt & $-9,23 \%$ & $-8,8$ Mio. Euro
\end{tabular}

anleihen, nimmt das Risiko weniger stark zu. Dennoch liegt die Rate für die Risikosteigerung immer noch bei mehr als $30 \%$.

\section{Konsequenzen und Ausblick}

Die Ergebnisse des letzten Kapitels zeigen, dass sich die Risiken der Portfolios deutlich unterscheiden, je nachdem welcher Zeitraum zum Schätzen der Parameter verwendet wird. Diese Änderungen des Risikos haben sowohl für institutionelle als auch private Investoren eine hohe Relevanz. Daher ist zu prüfen, wie dieser Effekt verringert werden kann. Es ist zu erkennen, dass die Korrelationen und Volatilitäten nicht ohne weiteres aus den historischen Daten geschätzt werden können, da teils erhebliche Schätzfehler auftreten. 
Tabelle 5

$\mathrm{VaR}_{\text {stoch }}$ Institutionell

\begin{tabular}{lcc}
\hline Phase / VaR & In $\%$ & In Euro \\
\hline Gesamt & $-5,66 \%$ & $-5,5$ Mio. Euro \\
Dotcom & $-4,87 \%$ & $-4,8$ Mio. Euro \\
Finanzkrise & $-7,70 \%$ & $-7,4$ Mio. Euro \\
Bullenmarkt & $-5,61 \%$ & $-5,5$ Mio. Euro
\end{tabular}

Tabelle 6

VaR $_{\text {stoch }}$ Deka Zukunftsplan I

\begin{tabular}{lcc}
\hline Phase / VaR & In $\%$ & In Euro \\
\hline Gesamt & $-36,52 \%$ & $-30,6$ Mio. Euro \\
Dotcom & $-39,96 \%$ & $-32,9$ Mio. Euro \\
Finanzkrise & $-53,61 \%$ & $-41,5$ Mio. Euro \\
Bullenmarkt & $-22,29 \%$ & $-20,0$ Mio. Euro
\end{tabular}

Tabelle 7

$\mathrm{VaR}_{\text {stoch }}$ Deka-Euroland Balance TF

\begin{tabular}{lcc}
\hline Phase $/ \mathrm{VaR}_{\text {stoch }}$ & In $\%$ & In Euro \\
\hline Gesamt & $-23,19 \%$ & $-20,7$ Mio. Euro \\
Dotcom & $-25,44 \%$ & $-22,5$ Mio. Euro \\
Finanzkrise & $-28,96 \%$ & $-25,1$ Mio. Euro \\
Bullenmarkt & $-14,61 \%$ & $-13,6$ Mio. Euro
\end{tabular}




\section{Historische Mittelwerte für Korrelationen}

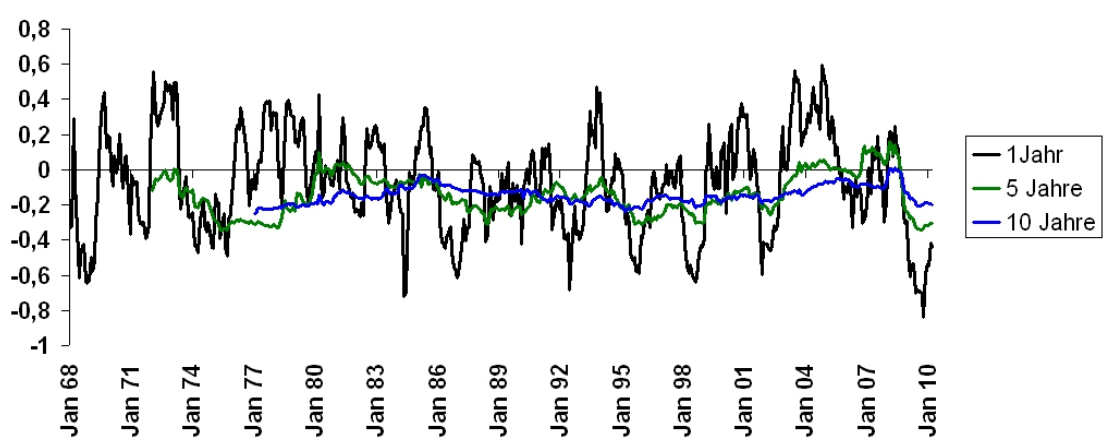

Abbildung 3: Gleitende Mittelwerte der Korrelation zwischen Staatsanleihen und Rohstoffen mit einer Historie von 1-Jahr, 5-Jahren und 10-Jahren.

Um diesen Effekt zu verdeutlichen, stellt Abbildung 3 unterschiedliche Verläufe von Schätzern der Korrelation zwischen Staatsanleihen und Rohstoffen dar. Dabei wurde die Korrelation aus historischen Daten geschätzt, wobei die Korrelation zu jedem Zeitpunkt aus den Daten des letzten Jahres, der letzten fünf Jahre bzw. der letzten zehn Jahre berechnet wurde.

Aus der Abbildung wird deutlich, dass die Schwankungen der Schätzer desto mehr abnehmen, je größer der für die Berechung verwendete Zeitraum ist. Folglich ergibt sich das Problem, dass eine lange Zeitreihe zu sehr unflexiblen Schätzern aufgrund einer starken Glättung des Ergebnisses führt. Daher werden Änderungen der Parameter erst nach sehr langer Zeit im Ergebnis berücksichtigt. Somit wird im Bullenmarkt das Risiko überund in den Krisen unterschätzt. Werden dagegen nur kurze Zeiträume in eine Schätzung einbezogen, so führt dies oft zu drastischen Schätzfehlern aufgrund der dadurch deutlich größeren Variabilität der Schätzer, insbesondere wenn zwei Phasen mit sehr unterschiedlichen Parametern direkt hintereinander folgen. So weicht der Schätzer der Korrelation unmittelbar nach dem Bullenmarkt deutlich von den wahren Werten ab, die in der Finanzkrise auftraten.

In der aktuellen Forschung gibt es verschiedene Ansätze mit sich ändernden Parametern umzugehen. So besteht die Möglichkeit moderne Parameterschätzer zu verwenden, die genauer und flexibler als der historische Mit- 
telwert sind. In diesem Zusammenhang sind insbesondere Zeitreihenmodelle interessant, da sie sich flexibel an die Änderungen der Datenstruktur anpassen. So berücksichtigen beispielsweise GARCH Modelle nicht nur die Historie der Zeitreihe, sondern auch ihre eigene Historie und die des Schätzfehlers. Ein Überblick hierzu findet sich z.B. in McNeil/Frey/Embrechts (2005).

Ein weiterer Ansatz zur frühzeitigen Erkennung von Parameteränderungen ist das Testen auf „Strukturbrüche“. Man spricht in der Statistik von Strukturbrüchen, wenn sich die Parameter, die die Zeitreihe definieren, ändern. In diesem Bereich wird aktuell intensive Forschung betrieben und es liegen erste vielversprechende Ergebnisse vor. Das frühzeitige Erkennen eines Strukturbruchs ermöglicht die rechtzeitige Einleitung von Maßnahmen, um auf die geänderte Marktsituation zu reagieren. Mehr zu Strukturbrüchen findet sich u.a. in Krämer/Tameze (2007), Qu/Perron (2007) und Krämer/van Kampen (2009).

\section{Danksagung}

Die Arbeit des ersten Autors wurde durch die DFG (Sonderforschungsbereichs 823) sowie durch das BMBF (Projekt INVERS) unterstützt, und die Arbeit des dritten Autors durch die Europäische Union, das Bundesministerium für Wirtschaft und Technologie, den ESF und EXIST unterstützt.

\section{Literatur}

[1] Andersen, T.G., Bollerslev, T., Diebold, F.X., Ebens, H. (2001): The distribution of realized stock return volatility, in: Journal of Financial Economics, Vol. 61, Nr. 1, S. 43-76

[2] Brinson, G. P., Hood, R., Beebower, G. L. (1986): Determinants of Portfolio Performance, in: Financial Analysts Journal, Vol. 42, Nr. 4, S. $39-48$

[3] Brinson, G. P., Singer, B. D., Beebower, G. L. (1991): Determinants of Portfolio Performance II: An Update, in: Financial Analysts Journal, Vol. 47, Nr. 3, S. 40-48 
[4] Chopra, V. K., Ziemba, W. T.(1993): The Effects of Errors in Means, Variances, and Covariances on Optimal Portfolio Choice, in: The Journal of Portfolio Management, S. 6-11

[5] Frennberg, P., B. Hansson (1993): Testing the Random Walk Hypothesis on Swedish Stock Prices 1919-1990, in: Journal of Banking and Finance, Vol. 17, Nr. 1, S. 175-191

[6] Ibbotson, R.G., Kaplan, P.D.(2000): Does Asset Allocation Policy Explain 40, 90 or 100 Percent of Performance? in: Financial Analysts Journal, S. 26-33

[7] Kallberg, J.G., Ziemba, W.T. (1984): Mis-Specifications in Portfolio Selection Problems, in: Risk and Capital, Hrsg. G. Bamberg und K. Spremann, New York, S. 74-87

[8] Krämer, W., Tameze, B. (2007): Structural Change and Long Memory in the GARCH(1,1)-Model, in: Economics Letters 97, S. 17-23

[9] Krämer, W., van Kampen, M. (2009): A simple nonparametric test for structural change in joint tail probabilities, Preprint SFB 823, http://www.statistik.tu-dortmund.de/sfb823-dp2009.html

[10] Longin, F., Solnik B. (1995): Is the correlation in international equity returns constant: 1960-1990?, in: Journal of International Money and Finance, 14, S. 3-26

[11] Markowitz, H.M. (1952): Portfolio Selection, in: Journal of Finance, 7, Nr. 1, S. 77-91

[12] McNeil, A. J., Frey, R., Embrechts, P. (2005): Quantitative Risk Management: Concepts, Techniques and Tools, Princeton Series in Finance

[13] Meric, I., Meric, G. (1997): Co-Movement of European Equity Markets Before and After the 1987 Crash, in: Multinational Finance Journal, Vol. 1, Nr. 2, S. 137-152

[14] Otto, K. (2008): Strategische Asset Allocation auf Gesamtbankebene. in: Akademie Deutscher Genossenschaften (ADG), 27./28. November 2008

[15] Qu, Z., Perron, P. (2007): Estimating and testing structural changes in multivariate regressions, in: Econometrica 75, S. 459-502 
[16] Rey, D.M. (2000): Time-varying Stock Market Correlations and Correlation Breakdown, in: Schweizerische Gesellschaft für Finanzmarktforschung, Nr. 4, S. 387-412

[17] Schäfer, K., Zimmermann, P. (1998): Portfolio Selection und Schätzfehler bei den erwarteten Renditen - Ergebnisse für den deutschen Aktienmarkt, in: Finanzmarkt und Portfolio Management, 12. Jg., Nr. 2, S. 131-149

[18] Zimmermann, H., Drobetz, W., Oertmann, P. (2002): Global asset allocation, New Jersey: John Wiley \& Sons 

